\title{
Multiple population theory: The extreme helium population problem
}

\author{
Sukyoung K. Yi \\ Yonsei University, Department of Astronomy, Seoul 120-749, Korea \\ email: yi@yonsei.ac.kr
}

\begin{abstract}
The spreads in chemical abundances inferred by recent precision observations suggest that some or possibly all globular clusters can no longer be considered as simple stellar populations. The most striking case is $\omega$ Cen in the sense that its bluest main-sequence, despite its high metallicity, demands an extreme helium abundance of $Y \approx 0.4$. I focus on this issue of "the extreme helium population problem" in this review.
\end{abstract}

Keywords. stars: abundances, AGB and post-AGB, chemically peculiar, evolution HertzsprungRussell diagram, Population II, Galaxy: general, abundances, globular clusters, ISM: abundances

\section{Introduction}

Globular clusters may not be a robust example for simple stellar populations any more. Perhaps there is no such thing as simple stellar populations from the beginning. The classic globular clusters, such as $\omega$ Cen, NGC 2808, and NGC 1851, are now suspected to be composed of heterogeneous populations, and recent data from space-based telescopes with unprecedented resolving accuracy are hinting at a great fraction of Milky Way globular clusters being composite populations, at least chemically.

At the centre of these debates is $\omega$ Cen. It has long been known as a mysterious object. To begin with, this spectacular southern cluster is the most massive in the Milky Way, with some million solar masses. Its unusually broad red-giant branch (RGB) was found to indicate discrete multiple populations by the magnificent effort and insight of Lee et al. (1999), using a mere $0.9 \mathrm{~m}$ telescope. More recent work, with much superior instruments, unambiguously revealed the multiplicity of this giant cluster. Norris (2004) and Bedin et al. (2005) sequentially found that the multiplicity is evident not just on the red giant branch but also on the main sequence. To everyone's surprise, the bluest main sequence is too blue for the measured metallicity for $\omega$ Cen and is in fact more metal rich than the redder main-sequence stars (Piotto et al. 2005). If such a blue colour for such a metal-rich population is real, it unavoidably indicates the possibility of the scorchingly high helium abundance, $Y \approx 0.4$. The blue main-sequence population constitutes $30 \%$ by number (Bedin et al. 2004; Sollima et al. 2007) and thus is not something we can simply sweep under the carpet. If there is any good news in this apparent nightmare, the blue main-sequence population seems at least to be younger than the majority of the stars in this cluster, perhaps by a couple of billion years (Villanova et al. 2007).

Such an age range has significance for the horizontal branch morphology in this cluster. This and many other clusters exhibit an extended horizontal branch, and its origin has been a long-debated issue. Apparently, the same level of extreme helium inferred by the blue main-sequence can also explain the extreme blueness of the extended horizontal branch (Lee et al. 2005). If this prevails in other clusters as well, the hitherto mysterious origin for the extended horizontal branch may also be solved by the extreme helium content. 
Apparently many more clusters show multiple sequences, either on the main sequence and/or on the sub-giant branch (Piotto 2008, this volume), even though it is not yet clear whether such multiplicities are also to be interpreted as originating from an extreme helium content. More massive clusters tend to show multiple sequences more often, and interestingly the same trend is found for the extended horizontal branch (Recio-Blanco et al. 2006; Lee et al. 2007).

Extragalactic counterparts to $\omega$ Cen and the like may have been found in the giant elliptical galaxy M87 in the Virgo cluster (Sohn et al. 2006; Kaviraj et al. 2007). Using the Hubble Space Telescope Sohn et al. (2006) found that most of the massive globular clusters in M87 are UV-bright, despite their likely old ages, as if they have an extremely hot horizontal branch. Through an extensive test using the UV-focused population synthesis models of Yi (2003), Kaviraj et al. (2007) concluded that the UV strengths (a tracer of the horizontal-branch morphology) of these clusters are even stronger than that of $\omega$ Cen, by more than a magnitude. Whatever is causing the multiplicity in $\omega$ Cen seems to affect the M87 clusters even more greatly.

The existence of massive clusters showing various anomalies seems to corroborate the idea of these clusters originally being something of a different nature, for example, nucleated dwarf spheroidal galaxies (Lee et al. 1999).

All things considered, there appears to be a huge conspiracy. It is not yet clear whether the cause of the multiplicity of the main sequence is the cause of that of the horizontal branch. However, they all fit in a very sensible storyline. Although it ruins the old and naive concept of "simple stellar populations", multiplicity by itself is perhaps not a huge problem. The extreme helium abundance inferred by the blue main-sequence population is an exciting discovery to observers but a desperate-to-forget nightmare to theorists. I now discuss why this is so.

\section{Significance}

The significance of this issue is immense. First, obtaining an understanding how such an extreme helium abundance could be possible is an interesting challenge. It also influences the current age-dating techniques that are based on precise main-sequence fitting and on detailed horizontal-branch analysis. The seemingly settled issue of the identity of the second parameter of horizontal-branch morphology may enter a new stage with the not-so-new idea of variations in helium abundance, with a clearer understanding ot the helium enrichment processes. The endless debate on the origin of the UV-upturn found in bright elliptical galaxies may also find a new and compelling explanation with helium. Obvious too is the impact on the issue of the age of the universe, as globular clusters and bright elliptical galaxies are often considered the oldest stellar populations in the Universe.

\section{Observational facts and inferences}

Finding a solution to the case of $\omega$ Cen is only a beginning step, since other clusters show different constraints, but it would still be a good start. So I attempt to find a solution, adopting some of the most widely discussed channels.

Our simplified constraints are as follows.

- The age separation: the blue main-sequence subpopulation is 1-3 billion years younger than the red main-sequence subpopulation; i.e., $t(b M S) \approx t(r M S)-1-3$ Gyr (Lee et al. 2005; Stanford et al. 2007; Villanova 2007). I think the exact value is poorly constrained but for now adopt a value $\Delta t=1 \mathrm{Gyr}$. 
- The mass fraction: the number (and mass) fraction of the blue main-sequence subpopulation is roughly $30 \%$ (Bedin et al. 2004), i.e., $f(b M S)=0.3$. I will try to aim to find a solution that satisfies this. However, this may not place as strong a constraint as I assume, if the mass evolution of sub-populations is complex. I will discuss this in detail in $\S 6$.

- Discrete sub-populations: the main sequence and horizontal branch splits appear very sharp and discrete. Hence, a stochastic element in a solution to the extreme helium abundance cannot be dominant. Instead, it has to offer a process that leads to a clear prediction in helium abundance.

- The metal abundance: $Z(r M S)=0.001$ and $Z(b M S)=0.002$ (Piotto et al. 2005). The metallicity of the blue main-sequence stars is difficult to pin down due to their faintness and so is still uncertain. But it seems clear that it is higher than that of the red main-sequence stars.

- The helium abundance: the helium abundance of the blue main sequence subpopulation is $40 \%$ by mass, i.e., $Y=0.4$. In reality, the observed colour-magnitude diagram shows up to 5 sub-populations. But it is impossible to make a model that pins down all the sub-populations found. Hence, I approximate them into 2 sub-populations: the red main sequence, with an ordinary helium abundance, and the blue main sequence, with an extreme helium abundance. As I will discuss at the end, it is perhaps very important to remind ourselves repeatedly that the helium abundance has not been directly measured, but is only inferred from main-sequence fitting. Despite this, I take the helium abundance as given.

- The helium enrichment parameter: the helium and metal abundances together lead to an incredible value for the helium enrichment parameter, $\Delta Y / \Delta Z \approx 70$. Ordinary populations with ordinary stars yield $\Delta Y / \Delta Z \approx 2-3$, even for a wide range of stellar initial mass functions. Hence, this poses the most challenging problem of all. I will focus most of my tests on this issue.

- Other elements: spectroscopic measurements of carbon and nitrogen are available, i.e., $[C / M] \sim 0$ and $[N / M] \sim 1$ for the blue main sequence population. However, their accuracy appears not to be as good as one might hope for, and estimated errors (i.e. measurement significance) are not provided. It is already a daunting task to reproduce just the helium properties, and so I will only use this information as a reference.

\section{Asymptotic giant branch stars}

The most obvious candidate origin for such an extreme helium abundance is asymptotic giant branch (AGB) stars (e.g., Izzard et al. 2004; D'Antona et al. 2005, among many papers). Although there is quite a scatter in the predictions of chemical yields from the AGB, there is a consensus that these stars generate a copious amount of helium, but only a small amount of metals (e.g., Maeder 1992). This is good for our present purposes, since we do not just want to produce a lot of helium but want also to achieve a very high value of the helium enrichment parameter $\Delta Y / \Delta Z$. Supernovae, for comparison, produce too high a mass in metals to satisfy this constraint, although they are also good producers of helium. This is such a basic point that it does not require much elaboration, but it has recently been discussed in a quantitative matter by Choi \& Yi (2007).

AGB stars in a narrow mass range $\left(M \approx 5-6 M_{\odot}\right)$ indeed release ejecta with a high value of the helium enrichment parameter, equal to that we aim to achieve. So if a population receives the stellar mass ejecta mainly from asymptotic giant stars and little else, it is in principle possible to achieve such a high value of helium enrichment parameter. More massive stars would produce both metals and helium. Hence, an ad hoc scenario can be 
set up to maximise the impact of the asymptotic giants in terms of the helium enrichment parameter, with all the mass ejecta from massive stars (say $M>M_{\mathrm{esc}}$ where $M_{\mathrm{esc}} \sim 5-$ $10 M_{\odot}$ ) posited to escape the gravitational potential where subsequent star formation occurs. The effectiveness of this maximum AGB scenario has been discussed by a few groups (e.g., Karakas et al. 2006; Bekki et al. 2007), and Choi \& Yi (2008) performed a detailed calculation to check its viability.

Choi \& Yi (2008) adopted a toy model where the original gas reservoir does not accept any new gas infall from outside and the material ejected from massive stars above $M_{\mathrm{esc}}$ escape it, supposedly via supernova explosions, hence maximising the helium enrichment effect from AGB stars. It is plausible that the kinetic energy of the material ejected from supernova explosions equals the escape energy from such a small potential well. It is assumed that some fraction $(50-100 \%)$ of the initial gas is used to form the first population (the red main sequence) and the subsequent population (the blue main sequence) will be born from the remnant gas, mixed with the material ejected from the first stellar population. The abundance of the initial gas is assumed to be the abundance of the red main sequence population of $\omega$ Cen. If a higher fraction of the initial gas reservoir is used to build the first population, it would obviously result in a higher value of helium abundance and helium enrichment parameter for the second population, but only a small amount of gas becomes available for the second population to form out of.

If we do not adopt any constraint on the age difference between the red and blue mainsequence populations, we can achieve a very high helium abundance $(Y \approx 0.36$ which is almost as high as we aim to reach) and the maximum value of helium enrichment parameter of about 70, as we hoped for. In this case, the age difference is roughly $0.1 \mathrm{Gyr}$, and the second generation is virtually a pure recycling product of the first generation, consisting of material ejected from stars within a narrow mass range of $5-6 M_{\odot}$. But in this case, the total mass ratio between the red and blue main-sequence populations becomes 99.3 : 0.7 ; that is, only $0.7 \%$ of the total population in $\omega$ Cen can benefit from this scenario. Since the blue main sequence population is observed to be $30 \%$ instead $0.7 \%$, there is a factor of 40 discrepancy! I call this "the mass deficit problem".

One may achieve somewhat different estimates by adopting different yields. For example, Renzini (2008) uses the recent yield for the so-called "super-AGB stars" to find that the mass discrepancy can be as small as 15 instead of 40 .

If we take the age difference of roughly $1 \mathrm{Gyr}$ as a valid constraint, the situation becomes dramatically worse. This is because, even if we assume the $M_{\mathrm{esc}}$ argument, the stars in the mass range $2-5 M_{\odot}$ will now contribute to the gas reservoir through stellar mass ejecta, with this ejecta in general of substantially lower helium abundance $(\sim 0.3)$ and helium enrichment parameter $(\sim 2-5)$. Consequently, this scenario with 1 Gyr age separation can achieve only up to $Y \approx 0.3$ and $\Delta Y / \Delta Z \approx 10$, while the upper limit in the mass fraction of the second generation is just $7 \%$ (instead of $30 \%$ ). Let alone the shortcoming in the helium properties, the mass fraction requirement cannot be met, either.

The verdict on the maximum AGB scenario and its variation can be summarised as follows. The extreme helium-related properties are almost impossible if the age difference is a meaningful constraint, hence making this scenario totally implausible. If the age separation constraint can be eased, then the extreme value of the helium enrichment parameter (but not the helium abundance itself) can be reproduced by the first generation of asymptotic giants, under the following conditions, and with the following criticisms.

- The stellar mass ejecta from massive stars of $M>6 M_{\odot}$ must all escape the gravitational potential well. If the 'super AGB' scenario (e.g. Siess 2007) is adopted, this mass limit can be as high as $10 M_{\odot}$. If all supernova ejecta leaves the system in a high- 
velocity wind, this is not a bad assumption, but assuming that the supernova ejecta leaves completely without affecting the remaining gas in the reservoir is extreme and very unlikely.

- The blue main-sequence population must form exactly $0.1 \mathrm{Gyr}$ after the red main sequence population, in disagreement with the $1 \mathrm{Gyr}$ separation suggested by previous studies. I personally think the age separation constraint is not very strong and thus $0.1 \mathrm{Gyr}$ is not particularly unappealing.

- The mass deficit of a factor of 40 (which can be somewhat smaller if 'super' AGB stars are adopted) is a serious threat and requires a rescue plan. A possible remedy may be found in the details of the cluster dynamical evolution, which is discussed in $\S 6$.

- An encouraging aspect of this scenario is that the discreteness of the separated populations is easy to explain. The second generation forms from the mass loss of the first generation, $0.1 \mathrm{Gyr}$ later.

\section{Fast-rotating massive stars}

A totally different solution was put forward by massive-star evolution models. Maeder \& Meynet (2006) suggested that metal-poor massive stars that are rotating nearly at their break-up speed may release a lot of helium via a slow wind before they start burning heavy elements and explode as a supernova. Their idea came from their earlier work (Maeder \& Meynet 2001) that suggested (1) low-metallicity stars reach break-up rotational speed more easily by the combined effects of stellar (slow) winds and rotation, (2) they have efficient mixing of their core materials, that is, helium and other heavier elements (depending on the rotation speed) are mixed out to the surface, and (3) during their blue loop, after the red giant phase, a fast contraction leads to extensive mass loss from the helium- and nitrogen-enhanced surface material.

The elemental yields via slow (stellar) winds are sensitive to the rotational speed adopted. For example for a $60 M_{\odot}$ star with $\log Z=-5$, a fast rotating model at $85 \%$ of the break-up speed yields the helium abundance of 5.86 solar mass, the metal abundance of 0.09 solar mass, and thus the helium enrichment parameter of 63.3 (which is very close to our aim!). On the other hand, for a moderately fast rotating model at $35 \%$ of the break-up speed, the yields become $\Delta Y=1.73, \Delta Z=2.6 e-5$, and $\Delta Y / \Delta Z \approx 10^{5}$. These extremely fast-rotating stars generate excessively high values of $\Delta Y / \Delta Z$ and too little of helium. The fast rotating stars overproduce carbon and nitrogen abundances compared to observation, while the moderate rotating stars reproduce the observation better. But we still select the fast-rotating models in our exercise (Choi \& Yi 2007) because they produce much more helium and thus are more likely to satisfy our aim.

The toy model of Choi \& Yi (2007), using the metal-poor massive rotating stars of Maeder \& Meynet (2006), shows that a simple population based on an ordinary initial mass function can indeed achieve high values of both the helium abundance and $\Delta Y / \Delta Z$ in the stellar mass loss, as we aim to recover. These values are further elevated by the helium-dominant contribution from asymptotic giants, until lower-mass giants become the main source of chemical yields. Thus this phenomenon of high helium properties lasts only for a short period of time, of order 0.1-0.2 Gyr, just as in the AGB scenario. Once the population becomes older than that, its accumulated stellar mass loss will no longer have such high values of its helium properties.

We find, however, that the amount of gas with these high helium properties can be only roughly $1.4 \%$ of the total stellar mass of $\omega$ Cen, which is a factor of 20 too small for it to be the sole solution to this problem. This mass deficit of a factor of 20 is smaller (and thus better) than that of the asymptotic giant branch star scenario simply because this 
time we have helium contributions from massive rotating stars as well as from asymptotic giants. Here, we assume that only the slow-wind material (stellar mass loss) from the massive stars remains in the gravitating system and the fast-wind material (explosions) leaves the system without polluting the gas reservoir.

In conclusion, we could not find a solution if the age separation of 1 Gyr or so is a meaningful constraint. For a much smaller age separation, of order $0.1 \mathrm{Gyr}$, we could achieve high values of the helium parameters, but even in this case the mass available for the formation of the second generation is a factor of 20 smaller than the requirements in $\omega$ Cen. This problem has been noted also by a much more detailed dynamical simulation of Decressin, Baumgardt, \& Kroupa (2008; see also the article by Decressin in this volume). We will discuss this further in $\S 6$.

Another serious problem in this scenario is the carbon abundance. While it depends strongly on the rotational speed adopted, the $60 M_{\odot}$ model, with $85 \%$ of the break-up speed, suggests that the slow-wind mass loss will be highly enriched in carbon, which is not supported by the observational data (Piotto et al. 2005).

For this scenario to be appealing, we also need to understand how a specific rotation speed is determined for the stars. Why does it happen to some clusters (like $\omega$ Cen) but not to others? Is it randomly given to each cluster, and not to each forming star? That will be very odd. This scenario with fast-rotating massive stars certainly adds to what was already possible from the asymptotic giant stars and thus provides a positive contribution. However, it alone does not appear to provide a full solution to our problem.

\section{Dynamical evolution}

The blue main-sequence population seems to be more centrally concentrated than is the red main-sequence population. If this were true from the start, one would expect that the spatially more-extended red main-sequence population would lose more stars throughout its dynamical evolution. D'Ercole et al. (2008; and also the poster at this meeting) indeed suggested that a substantial fraction of the first generation of stars may escape the system if some conditions are met. For example, if the initial mass distribution within each globular cluster follows the King profile and if its King radius is equal to its true tidal radius, then it is very easy to shed some high-velocity stars into space. In this case, only $2-3 \%$ of the original first-generation stars may remain in the cluster, mainly due to kinetic energy injection by supernova explosions and two-body relaxation. If this is true, it makes both the AGB scenario and the massive fast-rotating star scenario viable.

Whether these conditions were easy to meet by the first generation clusters is not yet clear, however. More traditional studies (e.g., Fall \& Zhang 2001) based on evaporation by two-body relaxation, gravitational shocks, and stellar mass loss suggest an order of magnitude milder mass evolution.

The mass evaporation is supposed to be sensitive to the mass of the cluster in the sense that a more massive cluster would shed less mass. So, if the dynamical evaporation was indeed the key to this extreme helium phenomenon, it would be very unlikely to happen preferentially to the most massive clusters. Unfortunately for this scenario, $\omega$ Cen is the Milky Way's most massive cluster and the other clusters showing multiplicity, NGC 2808 and M54, are among the most massive, too. Besides, the extended horizontal-branch globular clusters in the Milky Way, and the UV-brightest clusters in M 87, all occupy the highest-mass end in the total cluster-mass distribution of the host galaxy. In this sense, the dynamical evaporation picture loses its charm.

If D'Ercole et al.'s dramatic mass evolution is applicable to all globular clusters, then it would have a significant impact on the cluster luminosity-function evolution. Typical 
clusters in the Milky Way are of a million solar masses presently, which is in the same order as the mass of the giant molecular clouds, the main site of star formation, and also as the mass of the star clusters forming in nearby merging galaxies. In this regard, I feel that this scenario of shedding $98 \%$ of the initial mass of the first population is likely a rare event. Perhaps this is why the main sequence splits are not a common feature. Otherwise, that is, if such a dramatic mass evaporation had been true to all clusters, then we should find our galactic stellar halo to have at least ten billion solar masses, which is an order or magnitude greater than the current estimate. I strongly feel that physical understanding of the dynamical process (when such conditions are met) is required, and detailed dynamical models, cross-checking with the observed cluster luminosity functions, are called for.

\section{The first stars}

There must have been stars that formed before population I and II stars. This is evident from several arguments. Theoretically, the mass of the first objects that experience dynamical instability is estimated to be stellar rather than galactic. This is consistent with the fact that reionisation is (although indirectly) observed through the cosmic microwave background radiation studies. Observationally, despite the fact that the big bang itself did not generate any appreciable amount of heavy elements, totally metal-deficient stars are not found anywhere. Even the most metal-deficient stars show $\log Z / Z_{\odot} \sim-5$ and more typical metal-poor stars have metallicities greater than a hundredth of the solar value. This means that the pregalactic gas reservoir must have been substantially enriched in metals. The most probable objects for this are the first stars, a.k.a. population III stars. The first stars are often thought to have been very massive, above a hundred solar mass, while other possibilities are also being considered.

The duration of the first star-formation episode is considered to be extended well into that of population II (Bromm \& Loab 2006). If we are considering a proto-galactic scale system, the mixing timescale for the chemical elements may have been of order a hundred million years, and thus considering both the extended star formation timescale and varying mixing timescale, some chemical inhomogeneity in the gas reservoir for the population II star formation was inevitable.

Marigo et al. (2003) have computed the chemical yields for such metal-free stars of mass between 100 and $1000 M_{\odot}$. Surprisingly, their models suggest that the first stars were very efficient in generating and releasing helium into space, but not metals. This is mainly because the first stars had such an enormous radiation pressure that the balance between mass accretion and radiative pressure was difficult to achieve; that is, the strong radiation pressure blew away the gas that was being accreted. So the first energy generation involving hydrogen burning was possible, but before the star reaches the next stage it would release much materials processed by then: i.e., helium. This results in a high helium to metal ratio, as we were looking for.

Choi \& Yi (2007) have indeed investigated this effect on the helium enrichment in the gas cloud. They found that the the range between 100 and 1000 solar masses, a lowermass first star produces a much larger value of $\Delta Y / \Delta Z \sim 10^{7-8}$. (No, this is not a typo.) The first stars of $1000 M_{\odot}$ are predicted by this model to have $\Delta Y / \Delta Z \sim 10^{2}$, which is much closer to our aim. Adopting a Salpeter initial mass function $\dagger$, we found that a first-star population with a mass range $100-1000 M_{\odot}$ releases virtually no metals but

$\dagger$ As I type this part I just learned of Professor Salpeter's death. We have just lost one of the greatest astrophysicists of our time. 
abundant helium, and thus reaches $\Delta Y / \Delta Z \sim 500$. A population with a higher value of the lower mass bound results in a gradually lower value. Eventually, a population purely made up of 1000 solar-mass stars would have $\Delta Y / \Delta Z \approx 70$.

After letting the first star population evolve for a billion years the remnant gas cloud (primordial gas left out of the first star formation plus the stellar mass loss mixed evenly) reaches the metallicity of the blue main sequence $(Z=0.002)$, the helium abundance $(Y=0.4)$, and so the helium enrichment parameter $(\Delta Y / \Delta Z \approx 70)$, with no further free parameter.

This scenario is briefly investigated by Choi \& Yi (2008) and can be chronologically described as follows.

(a) The majority of first stars form in the Universe at redshift roughly at $20(t \equiv 0)$.

(b) These stars release much helium and some metals.

(c) The chemical mixing in the proto-galactic cloud took a long time, and after hundreds of millions of years, chemically mixed regions are more common than unmixed regions.

(d) From a chemically mixed region, the red main-sequence population of $\omega$ Cen forms $(t \sim 0.5 \mathrm{Gyr})$.

(e) From the pristine (unmixed) gas in the vicinity a second generation of first stars forms $(t \sim 0.7 \mathrm{Gyr})$.

$(f)$ They generate abundant helium and little metals and enrich the remnant gas cloud to $Z \sim 0.002, Y \sim 0.4$ and thus $\Delta Y / \Delta Z \sim 70$.

(g) From this gas cloud, the blue main-sequence population of $\omega$ Cen forms $(t \sim 1.5$ Gyr $)$.

(h) The blue main-sequence population merges into the more massive red main-sequence population soon after their formation.

This picture is very rough however and contains many caveats:

- The first-star chemical yields may be highly uncertain. A more robust understanding of the formation and evolution of the first stars will perhaps come in the near future, but, more importantly, independent calculations (besides Marigo et al.) are required immediately.

- In this scenario the first stars (at least the ones that led to the gas reservoir for the formation of the blue main-sequence population) should have very high mass, of order $1000 M_{\odot}$. This is not supported by some recent first-star studies.

- We need not just a couple of first stars in this region but more than one hundred. How such material gathers up in this proto cloud is a mystery, especially when first stars are often believed to form isolated, rather than in multiplets.

- The physics, in terms of the chemical mixing and its timescale, is highly uncertain, as is the case for other scenarios.

Given all these uncertainties, it is difficult to argue that the first-star scenario is any more compelling than others. However, it is still a very exciting possibility. After all, we astronomers are always the first one to find something wrong, as well as new and important. This conjecture at least implores for more studies on the first stars.

\section{Alternative theories}

Alternative theories are also available. The velocity-dispersion dependent surface pollution of AGB ejecta scenario was put forward by Tsujimoto et al. (2007). A similar surface-pollution scenario was presented by Newsham \& Terndrup (2007). While the channels for the pollution can be several, it provides an interesting possibility that the blue main-sequence stars are not truly so helium-rich as we believe, but pretend to be so by having unusually high helium abundance only on the stellar surface. Mass transfer 
of the surface material in binary stellar systems could be one channel, or if stars pass through the central region of the cluster, where helium-rich gas from the accumulated stellar mass loss is located, such stars may be polluted on the surface. However, it is very unlikely that $30 \%$ of the stars get contaminated like this. Besides, all these processes would occur in a random manner, so that the discreteness of the blue main-sequence would be unnatural.

The possibility of having primordial fluctuations in the helium density was presented by Chuzhoy (2006). In this study, the helium diffusion timescale for primordial gas of stellar mass was of order a hundred million years, and thus some of the birth clouds for the first stars were heavily enriched in helium. But again, the diffusion timescale must depend on the conditions in each birthcloud, which should be rather random, which again makes the main-sequence discreteness a tough problem to solve.

\section{Conclusions}

Theorists are often very optimistic, thinking that a tough problem to solve is challenging instead of mind-boggling. But I must admit that I am much more than puzzled by this "extreme helium population problem". The presence of multiple populations in globular-cluster size populations is surely a problem, given that numerical simulations of the kind performed by Bate, Bonnell, \& Bromm (2008) suggest that the star formation in a cluster probably happens on the crossing time scale, which is only on the order of a million years. But we have seen other small populations that have a complex star formation history, e.g., the Carina dwarf galaxy (Smecker-Hane et al. 1994). A more critical issue is the extreme value of the helium properties. I do not believe that we have a compelling theory yet. Asymptotic giant branch stars are a familiar class and thus makes our mind susceptible. But I believe that I have shown that AGB-models still have a mass deficit problem, by a factor of at least 40 , which is threateningly large even to astronomers. The same is true for the scenario of massive stars rotating nearly at the break-up speed. They alone cannot provide the full answer and suffer from a similar mass deficit problem. Its physical plausibility is also something to be worked out. The first-star scenario is fascinating because first stars are a mystery in general. We believe that they were once around but have never seen them, a bit like black holes. They provide a plausible solution, but just barely. It has so many caveats and uncertainties that cannot be clarified in the next few years. Hence it loses its charm, too.

I said at the end of my presentation at this conference that the enigmatic extreme helium population is so tough to theorists that I would almost feel happy if someone comes up to say "It was all a mistake from the start. There is no such extreme helium population". George Meynet disagreed with me. He instead said the problem is so enigmatic that we are greatly challenged and excited. I became humble at his constructive attitudes. I hope to see a more believable solution in the near future.

\section{Acknowledgements}

I thank Ena Choi for countless constructive discussions. Much of this review is based on several key papers written by Choi \& Yi (2007, 2008), Decressin, Charbonnel \& Meynet (2008), and by Renzini (2008). I am grateful to Young-Wook Lee, Suk-Jin Yoon, Ken Nomoto (during my visit to Tokyo University), and Enrico Vesperini for constructive discussions. Special thanks are due to Changbom Park for stimulating discussion on stellar collisions in clusters during my visit to the KIAS. This research has been supported by Korea Research Foundation (SKY). 


\section{References}

Bedin, L. R., Piotto, G., Anderson, J., Cassisi, S., King, I. R., Momany, Y., \& Carraro, G. 2004, ApJ, 605, L125

Bekki, K., Campbell, S. W., Lattanzio, J. C. \& Norris, J. E., 2007, MNRAS, 337, 335

Choi, E. \& Yi, S. K. 2007, MNRAS, 375, L1

Choi, E. \& Yi, S. K. 2008, MNRAS, 386, 1332

Chuzhoy, L. 2006, MNRAS, 369, L52

D’Antona, F., Bellazzini, M., Caloi, V., Pecci, F. F., Galleti, S., \& Rood, R. T. 2005, ApJ, 631, 868

Decressin, T., Charbonnel, C., \& Meynet, G. 2007, A\&A, 475, 859

Decressin, T., Baumgardt, H., \& Kroupa, P. 2008, $A \mathscr{E} A$, in press

Fall, S. M. \& Zhang, Q. 2001, ApJ, 561, 751

Izzard, R. G., Tout, C. A., Karakas, A., \& Pols, O. R. 2004, MNRAS, 350, 407

Karakas, A. I., Fenner, Y., Sills, A., Campbell, S. W., \& Lattanzio, J. C. 2006, ApJ, 652, 1240

Kaviraj, S., Sohn, S. T., O'Connell, R. W., Yoon, S.-J, Lee, Y.-W., \& Yi, S. K. 2007, MNRAS, 377,987

Lee, Y.-W., Joo, J.-M., Sohn, Y.-J., Rey, S.-C., Lee, H.-C., \& Walker, A. R. 1999, Nature, 402, 55

Lee, Y.-W., Joo, S.-J., Han, S.-I., Chung, C., Ree, C. H., Sohn, Y.-J., Kim, Y.-C., Yoon, S.-J., Yi, S. K., \& Demarque, P. 2005, ApJ, 621, L57

Lee, Y.-W., Gim, H. B., \& Casetti-Dinescu, D. I. 2007, ApJ, 661, L49

Maeder, A. 1992, A\& $A, 264,105$

Maeder, A. \& Meynet G. 2001, $A \mathscr{E} A, 373,555$

Maeder, A. \& Meynet G. 2006, A\& A, 448, L37

Newsham, G. \& Terndrop, D. M. 2007, ApJ, 664, 332

Norris, J. E. 2004, ApJ, 612, 25

Piotto et al. 2005, ApJ, 621, 777

Piotto, G., Bedin, L. R., Anderson, J., King, I. R., Cassisi, S., Milone, A. P., Villanova, S., Pietrinferni, A., \& Renzini, A. 2007, ApJ, 661, 53

Recio-Blanco, A., Aparicio, A., Piotto, G., de Angeli, F., \& Djorgovski, S. G. 2006, A $\& A, 452$, 875

Renzini, A. 2008, MNRAS, 391, 354

Siess, L. 2007, A\&A, 476, 893

Smecker-Hane, T. A., Stetson, P. B., Hesser, J. E., \& Lehnert, M. D. 1994, AJ, 108, 507

Sohn, S. T., O'Connell, R. W., Jundu, A., Landsman, W. B., Burstein, D., Bohlin, R. C., Frogel, J. A., \& Rose, J. A. 2006, AJ, 131, 866

Sollima, A., Ferraro, F. R., Ballazzini, M., Origlia, L., Straniero, O., \& Pancino, E. 2007, ApJ, 654,915

Stanford, L. M., Da Costa, G. S., Norris, J. E., \& Cannon, R. D. 2007, ApJ, 667, 911

Tsujimoto, T., Shigeyama, T., \& Suda, T. 2007, ApJ, 654, 139

Villanova, S., Piotto, G., King, I. R., Anderson, J., Bedin, L. R., Gratton, R. G., Cassisi, S., Momany, Y., Bellini, A., Cool, A. M. Recio-Blanco, A., \& Renzini, A. 2007, ApJ, 663, 296

Yi, S. 2003, ApJ, 582, 202

\section{Discussion}

M. Pinsonneault: We don't see evidence of high helium in field populations. Is this consistent with the high He models, or do they pose constraints?

S. YI: That's a very interesting test. But, according to both the AGB scenario and the massive rotating star scenario, the age separation between the first and second generations must be in a very narrow window (i.e., $\sim 30-300 \mathrm{Myr}$ ). Unless this condition is met, the extreme helium population may not be possible, which would explain the paucity of them in the field. 
R. WYSE: Does the assumption that the clusters can retain the ejecta of stars with $M \sim 6 M_{\odot}$ (your escape mass) have implications for the gas and dust content of globular clusters (there are very low upper limits)?

S. Yi: You're right. A more acceptable $M_{\text {esc }}$ would be close to $10 M_{\odot}$. A model with $M_{\mathrm{esc}}=6$ is an extreme one, and it tells us how extreme the AGB scenarios must be to make them work.

J. Melbourne: I was wondering what is driving the blue MS colors, is it an age effect or is the helium playing a role?

S. YI: Extreme helium abundance.

G. Meynet: The Na-O anticorrelation is not seen in halo field stars. In the framework of the Pop III scenario that you presented, how do you explain the absence in field stars? Why only in clusters?

S. Yi: Yes, if there is a causal connection between the Na-O anti-correlation and the extreme helium, that would be a threat to the first-star scenario. 
S. K. Yi

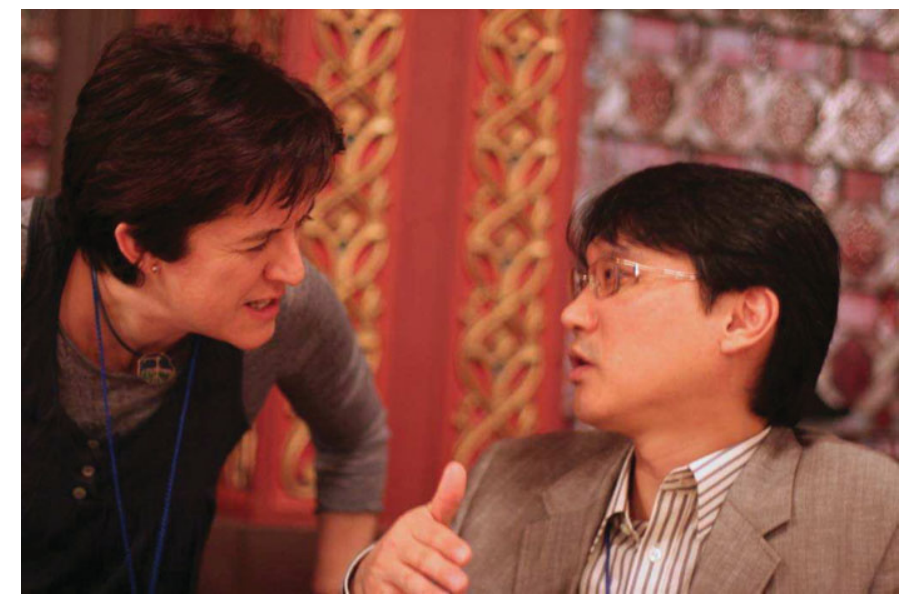

Carme Gallart and Sukyoung Yi

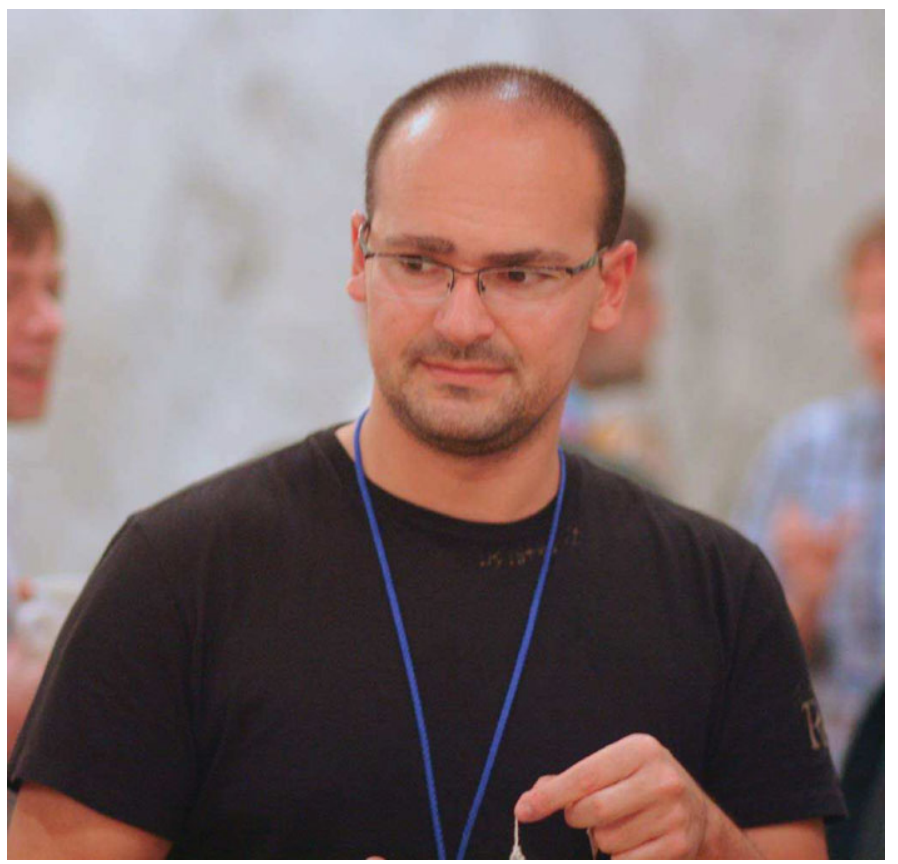

Michelle Cignoni 